\title{
Optically active hydrogen dimers in silicon
}

\author{
B. Hourahine ${ }^{\dagger}$ and R. Jones \\ School of Physics, The University of Exeter, Exeter EX4 4QL, UK \\ A. N. Safonov \\ University of Durham, South Road, Durham, DH1 3LE, UK \\ S. Öberg \\ Department of Mathematics, University of Luleå, Luleå S-97187, Sweden \\ P. R. Briddon \\ Department of Physics, The University of Newcastle upon Tyne, \\ Newcastle upon Tyne NE1 7RU, United Kingdom \\ S. K. Estreicher \\ Department of Physics, Texas Tech University, Lubbock, Texas 79409-1051, USA
}

\begin{abstract}
First principles calculations are used to explore the structure and properties of several defects which are prominent luminescent centers in Si. The trigonal defects $B_{41}$ and $B \frac{1}{71}$, which are known to contain two hydrogen atoms in equivalent and inequivalent sites respectively, are attributed to a hexavacancy containing two $\mathrm{H}$ atoms in different configurations. It is suggested that the $J$ luminescence centers arises from a stable hexavacancy without hydrogen atoms.
\end{abstract}

Hydrogen dimers were first suggested to exist in crystalline silicon in the form of molecules $[1,2]$ sited at tetrahedral interstitial lattice-positions. These molecules have subsequently been observed in hydrogen-soaked [3] and low-temperature plasma-treated silicon [4] (the substrate is held at $\leq 150^{\circ} \mathrm{C}$ during exposure). Higher temperature plasma-treatment instead forms hydrogen molecules inside platelet or void-like structures in the subsurface region of the silicon $[4,5]$.

There are several other di-hydrogen and multihydrogen defects known to form complexes with native defects in implanted or irradiated silicon. A large number of vacancy-hydrogen complexes of the form $\mathrm{V}_{m} \mathrm{H}_{n}$ $m=1, n=1 \cdots 4 ; m=2, n=1$ or 6 , and $m=3$ or 4 with $n=1$ have been identified in proton-implanted silicon $[6,7]$.

Similarly, electron paramagnetic investigation of multivacancy centers in $\mathrm{Si}$ have identified $\mathrm{V}_{1}, \mathrm{~V}_{2}$ [8], $\mathrm{V}_{3}, \mathrm{~V}_{4}$ and $\mathrm{V}_{5}[9]$. The last has been correlated with the $P 1$ center and is a non-planar defect with $C_{1 h}$ symmetry. The larger vacancy centers are formed in irradiated material when subjected to a heat treatment. Thus $\mathrm{V}_{5}$ is formed around $170{ }^{\circ} \mathrm{C}$ and is stable until $\sim 450{ }^{\circ} \mathrm{C}$.

Photoluminescence (PL) has revealed a large number of centers in silicon which has been treated by in-diffusion of hydrogen at high temperatures with a subsequent irradiation and heat treatment [10]. These centers were produced by irradiation with $6 \times 10^{17} \mathrm{~cm}^{-2}$ electrons, or $10^{17} \mathrm{~cm}^{-2}$ thermal neutrons, of silicon which has been soaked in hydrogen, followed by annealing at $\sim 450{ }^{\circ} \mathrm{C}$.
Recent Zeeman and uniaxial studies demonstrate that the PL centers which are produced by irradiation bind an exciton consisting of a electron in a deep $(-/ 0)$ level near $E_{c}$ with a loosely bound hole $[11,12]$. These defects have labels of the form $B_{y z}^{x}$, which specifies the exciton binding energy associated with the strongest PL line as $x y . z \mathrm{meV}$. So for example $B \frac{1}{71}$ has an exciton binding energy of $17.1 \mathrm{meV}$, and a strong PL line at $1.138 \mathrm{eV}$. In addition some of the transitions have specific names, such as the family of $J$-lines which are different exciton states of the $B_{80}^{4}$ center giving a luminescence at around $1.108 \mathrm{eV}[13,14]$.

Studies using $\mathrm{H}$ and $\mathrm{D}$ mixtures demonstrated that some of these centers contain two hydrogen atoms. Zeeman splitting studies have shown that $B_{80}^{4}, B_{41}$, and $B{ }_{71}^{1}$ possess trigonal symmetry. In the absence of experiments performed in an electric field, the question of whether the defects have a center of inversion has not been resolved. $B_{80}^{4}$ does not possess any hydrogen atoms; $B{ }_{71}^{1}$ contains two inequivalent hydrogen atoms, and $B_{41}$ possesses two equivalent hydrogen atoms. The high symmetry of these centers strongly restricts possible structures of the defects, particularly in the case of $B_{41}$, which although it is usually reported as possessing $C_{3 v}$ symmetry, actually requires the higher $D_{3 d}, D_{3}$ or $C_{3 i}$ symmetries to give equivalent sites for the two hydrogen atoms.

There are few defect structures with such high symmetry in silicon, since the complex must possess a principle three-fold rotational axis along one of the $\langle 111\rangle$ direc-

$\dagger$ Electronic address: bh@excc.ex.ac.uk 
tions. If the defect also possesses inversional symmetry as required by the $D_{3 d}$ and $C_{3 i}$ point-groups, or three coplanar two-fold rotation axes as required by $D_{3}$, then here are only two sites in the diamond lattice with such high symmetry at which the defect can sit. These are the bond-center and hexagonal lattice sites. The obvious structure, consisting of two anti-bonded hydrogen atoms attached to the pair of silicon atoms surrounding a bond centered site must be discounted, since such a defects should be stable only at low temperatures.

The combined requirements of creation by irradiation and high thermal stability point towards a multivacancyhydrogen complex. A plausible candidate is a complex between $\mathrm{V}_{6}$ and hydrogen.

$\mathrm{V}_{6}$ is expected to form in irradiated material which is heated. If the material contains hydrogen molecules these will readily react with $\mathrm{V}_{6}$ to form dimer structures, which in turn suggests that the hydrogen free $B_{80}^{4}$ defect should be $\mathrm{V}_{6}$ itself. This is consistent with the strong preference this defect displays to align along [111] stress during formation [14], which is indicative of a vacancy center. Further more, this center appears to react with hydrogen to form the $B_{41}$ and $B_{71}^{1}$ [15].

The defects considered were each constructed in a $\mathrm{Si}_{154} \mathrm{H}_{108}$ cluster centered on the hexagonal site. The wave-function basis consisted of independent $s$ and $p$ Gaussian orbitals, with either four different exponents sited at each $\mathrm{Si}$ atom, or three at the $\mathrm{H}$ atoms of the dimers. A fixed linear combination of two Gaussian orbitals was sited on the $\mathrm{H}$ atoms which terminated the cluster. In addition, two Gaussian functions were placed across every $\mathrm{Si}-\mathrm{Si}$ bond and the $\mathrm{Si}-\mathrm{H}$ bonds the defects. The charge density was fitted with five independent Gaussian functions with different widths on each Si atom, and four (three) on the central (terminating) $\mathrm{H}$ atoms. Two extra Gaussian functions were placed at each bond center. All atoms, including the terminating $\mathrm{H}$ ones, were allowed to relax by a conjugate gradient method. The second derivatives of the energy were found for the $\mathrm{H}$ atoms and the $\mathrm{Si}$ atoms they were bonded to. Further details of the spin-polarized LDF method can be found in Ref. [16].

We firstly simulate $V_{6}$ itself by removing the six atoms nearest to the hexagonal site. The resulting structure possesses $D_{3 d}$ symmetry and strongly reconstructs on relaxation to form six new bonds of length $2.60 \AA$ between the twelve dangling bonds as shown in Fig. 1a. The resulting structure and electronic Kohn-Sham levels, display a well defined band-gap, which are consistent with previous calculations [17]. This defect possibly possesses states in the gap very near the conduction band, but it is difficult to decide whether such near-conduction levels are localized on the defect in cluster calculations. The character of the deepest of these shallow states is $a_{1 g}$, and is anti-bonding to both the axial $\mathrm{Si}-\mathrm{Si}$ bonds and the six reconstructed bonds. If the defect is identified with $B_{80}^{4}$, then this state corresponds to the $(-/ 0) \Gamma_{1}$ level observed experimentally [11]. However, experimental measurement of the effect of an electrical field on this state would be required to confirm the proposed inversionally symmetric defect.

There are several potential models for $B_{41}$ based on $\mathrm{V}_{6}$, but all these require the hydrogen atoms to be sited at equivalent positions on the three-fold axis of the defect. One possible model would be a hydrogen molecule aligned along [111] and sited at the center of inversion of the defect. Alternately the $\mathrm{H}-\mathrm{H}$ bond could be broken and the atoms placed at anti-bonding sites to the axial $\mathrm{Si}-\mathrm{Si}$ bonds, as in Fig. 1b. A third possibility is that the hydrogen atoms are near the bond-centered sites within the axial Si-Si bonds. The second, anti-bonded structure is unstable and spontaneously relaxes back to the [111] aligned molecule structure. We calculate that the molecule itself is only metastable at the defect's center of inversion, and will dissociate with a barrier of $0.23 \mathrm{eV}$. It does this by breaking one of the reconstructed $\mathrm{Si}-\mathrm{Si}$ bonds shown in Fig. 1a, to form two $\mathrm{Si}-\mathrm{H}$ bonds, giving rise to a structure which is $1.76 \mathrm{eV}$ lower in energy and of $C_{1 h}$ symmetry as shown in Fig. 1c. The third structure causes a further reconstruction of $\mathrm{V}_{6}$, by breaking the two axial $\mathrm{Si}-\mathrm{Si}$ bonds and forming $\mathrm{Si}-\mathrm{H}$ bonds. The two dangling Si bonds thus formed pair together in the middle of the defect, leaving the structure with $D_{3 d}$ symmetry (shown in Fig. 1d). This reconstruction is energetically very favorable, being $0.80 \mathrm{eV}$ lower in energy than the $C_{1 h}$ structure in Fig. 1c. The KS levels of this defect again show evidence for a very shallow $(-/ 0)$ level, of symmetry $a_{1 g}$ consisting of a combination of anti-bonding states around the defect. There are two high frequency modes related to the hydrogen in this structure, of symmetries $A_{1 g}$ and $A_{2 u}$, which we find to lie at 2033 and $2021 \mathrm{~cm}^{-1}$ respectively. This structure posses properties consistent with $B_{41}$.

We now consider potential structures for $B_{71}^{1}$. Again this structure requires a $C_{3}$ axis, but due to the inequivalent hydrogen atoms the symmetry of the defect must be of lower order, i.e. $C_{3 v}$ or $C_{3}$. If it is assumed that dangling silicon bonds are energetically unfeasible, there is an obvious structure which can be derived from the bond-centered model for $B_{41}$. This defect consists of one hydrogen atom lying at a bond centered site, and the other atom directly passivating the silicon atom which is displaced by the bond-centered $\mathrm{H}$. There are two locations on either side of the silicon atom at which the hydrogen might be positioned. If the hydrogen were placed on the side nearest the other $\mathrm{H}$ atom, this would bring the two atoms into close proximity and thus raise the energy of the defect, this suggest that the hydrogen is sited on the opposite side of the silicon atom as shown in Fig. 1e. The relative energies of these defects are $0.13 \mathrm{eV}$ in favor of the structure with the hydrogen atoms on opposite sides of the silicon. The more stable structure, which is 
reminiscent of $\mathrm{H}_{2}^{*}[18]$, is $0.09 \mathrm{eV}$ higher in energy than the the $C_{1 h}$ defect formed by the dissociation a hydrogen molecule inside $\mathrm{V}_{6}$. The KS levels again show evidence of a near conduction-band $a_{1}$ state localized on the defect in both cases. Both of these models possess two high frequency $A_{1}$ vibrational modes, lying at 2149 and $2029 \mathrm{~cm}^{-1}$ for the structure with the two $\mathrm{H}$ atoms lying close together, or 2051 and $2010 \mathrm{~cm}^{-1}$ for the hydrogen on opposite sides of the silicon.

The dependence of the intensity of the PL due to $B_{80}^{4}$ demonstrates that the exciton is thermally bound with an energy of $18 \mathrm{meV}$ [19] and this can be taken to be the ionization energy of the hole. The exciton binding energy relative to a free electron and hole is the sum of $48.0 \mathrm{meV}$ and the free exciton binding energy of $14.3 \mathrm{meV}$. From these results, we place the $(-/ 0)$ level of $\mathrm{V}_{6}$ at $E_{c}-44.3 \mathrm{meV}$.

In conclusion, we have shown that complexes between $\mathrm{V}_{6}$ and hydrogen have properties consistent with those of the photoluminescent $B_{41}$ and $B_{71}^{1}$ centers formed in irradiated silicon. We also suggest the the $J$ family of PL defects are due to the hydrogen-free hexavacancy.

\section{ACKNOWLEDGMENTS}

S. Ö. thanks NFR and TFR for financial support. S. K. E. thanks the R. A. Welch Foundation (grant D1126) and NREL (contract XAD-7-17652-01). We also thank the ENDEASD network and E. V. Lavrov for helpful discussions.

\section{REFERENCES}

[1] A. Mainwood and A. M. Stoneham, Physica B \& C 116(1-3), 101 (1983).

[2] J. W. Corbett, S. N. Sahu, T. S. Shi, and L. C. Snyder, Phys. Lett. A 93(6), 303 (1983).

[3] R. E. Pritchard, M. J. Ashwin, J. H. Tucker, and R. C. Newman, Phys. Rev. B 57(24), 15048 (1998).

[4] A. W. R. Leitch, V. Alex, and J. Weber, Phys. Rev. Lett. 81(2), 421 (1998).

[5] B. Hourahine, R. Jones, S. Öberg, R. C. Newman, P. R. Briddon, and E. Roduner, Phys. Rev. B 57(20), 12666 (1998).

[6] B. Bech Nielsen, L. Hoffmann, and M. Budde, Mater. Sci. Eng. B 36(1-3), 259 (1996).

[7] P. Stallinga, P. Johannesen, S. Herstrom, K. Bond Nielsen, B. Bech Nielsen, and J. R. Byberg, Phys. Rev. B 58(7), 3842 (1998).

[8] G. D. Watkins, Deep centers in semiconductors (Gordon and Breach Science Publishers, Switzerland, 1992), chap. 3, 2nd ed.

[9] Y. H. Lee and J. W. Corbett, Phys. Rev. B 9(10), 4351 (1974).

[10] A. S. Kaminskii, E. V. Lavrov, V. A. Karasyuk, and M. L. W. Thewalt, Phys. Rev. B 50(11), 7338 (1994).

[11] A. N. Safonov, E. C. Lightowlers, and G. Davies, Phys. Rev. B 56(24), 15517 (1997).

[12] A. N. Safonov and E. C. Lightowlers, Mater. Sci. Eng. B 58(1-2), 39 (1999).

[13] R. Sauer and J. Weber, Physica B \& C 116(1-3), 195 (1983).

[14] A. S. Kaminskii and E. V. Lavrov, Solid State Commun. 106(11), 751 (1998).

[15] B. Hourahine, R. Jones, A. N. Safonov, S. Öberg, P. R. Briddon, and S. K. Estreicher, Identification of the hexavacancy in silicon with the $b_{80}^{4}$ optical center (1999), submitted to Phys. Rev. B.

[16] R. Jones and P. R. Briddon, The ab initio cluster method and the dynamics of defects in semiconductors (Academic Press, Boston, 1998), vol. 51A of Semiconductors and Semimetals, chap. 6.

[17] J. L. Hastings, S. K. Estreicher, and P. A. Fedders, Phys. Rev. B 56(16), 10215 (1997).

[18] B. Bech Nielsen, J. Holbech, R. Jones, P. Sitch, and S. Öberg, Mater. Sci. Forum 845-852, 143 (1994).

[19] A. S. Kaminskii, B. M. Leiferov, and A. N. Safonov, Sov. Phys. Solid State 29, 551 (1987). 
FIG. 1: Schematic diagrams of $\mathrm{V}_{6}$ and related defects. a) $\mathrm{V}_{6}$ displaying the six vacancy sites, and the subsequent reconstruction caused by them. b) $\mathrm{V}_{6}$ plus two anti-bonding hydrogen atoms. c) The structure formed by the dissociation of the $\mathrm{H}_{2}$ molecule, which breaks one of the reconstructed bonds. d) The proposed $B_{41}$ structure is shown with the two equivalent hydrogen atom that force the further reconstruction of the two axial silicon atoms across the center of the defect. e) The second proposed $B_{71}^{1}$ structure with the two inequivalent hydrogen atoms arranged as in $\mathrm{H}_{2}^{*}$.
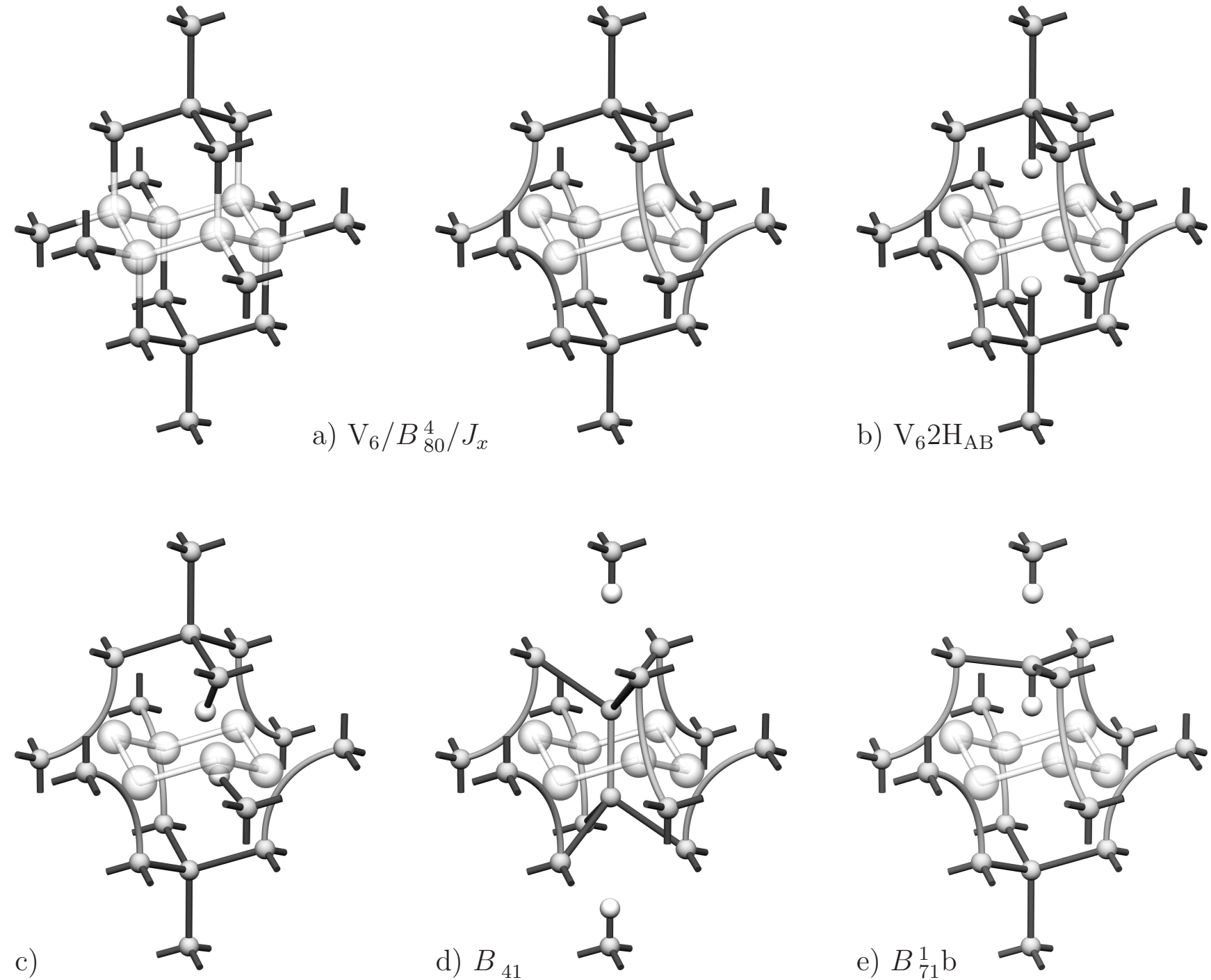